\title{
Nocturnal intermittent hypoxia and the development of type 2 diabetes: the Circulatory Risk in Communities Study (CIRCS)
}

\author{
I. Muraki $\cdot$ T. Tanigawa $\cdot$ K. Yamagishi $\cdot$ S. Sakurai $\cdot$ \\ T. Ohira $\cdot$ H. Imano $\cdot$ A. Kitamura $\cdot$ M. Kiyama • \\ S. Sato $\cdot$ T. Shimamoto $\cdot$ M. Konishi $\cdot$ H. Iso • \\ for the CIRCS Investigators
}

Received: 14 June 2009 / Accepted: 30 October 2009 /Published online: 28 November 2009

(C) Springer-Verlag 2009

\begin{abstract}
Aims/hypothesis Although the associations between obstructive sleep apnoea and type 2 diabetes mellitus have been reported in cross-sectional design studies, findings on the prospective association between the two conditions are limited. We examined prospectively the association between nocturnal intermittent hypoxia as a surrogate marker of obstructive sleep apnoea and risk of type 2 diabetes.

Methods A total of 4,398 community residents aged 40 to 69 years who had participated in sleep investigation studies between 2001 and 2005 were enrolled. Nocturnal inter-
\end{abstract}

I. Muraki $\cdot$ T. Ohira $\cdot$ H. Imano $\cdot H$. Iso $(\varangle)$

Public Health, Department of Social and Environmental Medicine,

Graduate School of Medicine, Osaka University,

2-2 Yamadaoka,

Suita, Osaka 565-0871, Japan

e-mail: iso@pbhel.med.osaka-u.ac.jp

I. Muraki $\cdot$ A. Kitamura $\cdot$ M. Kiyama $\cdot$ S. Sato $\cdot$ T. Shimamoto $\cdot$ M. Konishi

Osaka Medical Center for Health Science and Promotion,

Osaka, Japan

T. Tanigawa $\cdot$ S. Sakurai

Department of Public Health,

Doctoral Program in Social Medicine,

Graduate School of Medicine, Ehime University,

Toon, Japan

K. Yamagishi

Department of Public Health Medicine,

Graduate School of Comprehensive Human Sciences,

and Institute of Community Medicine, University of Tsukuba,

Tsukuba, Japan

S. Sato

Chiba Prefectural Institute of Public Health,

Chiba, Japan mittent hypoxia was assessed by pulse-oximetry and defined by the number of oxygen desaturation measurements $\leq 3 \%$ per $\mathrm{h}$, with five to $<15$ per $\mathrm{h}$ corresponding to mild and 15 events or more per $\mathrm{h}$ corresponding to moderate-to-severe nocturnal intermittent hypoxia, respectively. The development of type 2 diabetes was defined by: (1) fasting serum glucose $\geq 7.00 \mathrm{mmol} / \mathrm{l}(126 \mathrm{mg} / \mathrm{dl})$; (2) non-fasting serum glucose $\geq 11.1 \mathrm{mmol} / 1(200 \mathrm{mg} / \mathrm{dl})$; and/ or (3) initiation of glucose-lowering medication or insulin therapy. Multivariable model accounted for age, sex, BMI, smoking status, current alcohol intake, community, borderline type 2 diabetes, habitual snoring, excessive daytime sleepiness, sleep duration and (for women) menopausal status.

Results By the end of 2007, 92.2\% of participants had been followed up (median follow-up duration [interquartile range] 3.0 [2.9-4.0] years) and 210 persons identified as having developed diabetes. The multivariable-adjusted hazard ratio $(95 \% \mathrm{CI})$ for developing type 2 diabetes was 1.26 (0.91-1.76) among those with mild nocturnal intermittent hypoxia and 1.69 (1.04-2.76) among those with moderate-to-severe nocturnal intermittent hypoxia $(p=0.03$ for trend).

Conclusions/interpretation Nocturnal intermittent hypoxia was associated with increased risk of developing type 2 diabetes among middle-aged Japanese.

Keywords Intermittent hypoxia - Longitudinal studies . Sleep apnoea $\cdot$ Type 2 diabetes mellitus

$\begin{array}{ll}\text { Abbreviations } \\ \text { AHI } & \text { Apnoea-hypopnoea index } \\ 3 \% \mathrm{ODI} & 3 \% \text { Oxygen desaturation index } \\ \mathrm{OSA} & \text { Obstructive sleep apnoea } \\ \mathrm{RDI} & \text { Respiratory disturbance index } \\ \mathrm{SpO}_{2} & \text { Peripheral blood oxygen saturation }\end{array}$




\section{Introduction}

The increasing incidence of type 2 diabetes worldwide is a serious public health problem, because of the associated high risk of cardiovascular disease, renal failure and mortality. The worldwide prevalence of type 2 diabetes for all ages was $2.8 \%$ in 2000 and is estimated to be $4.4 \%$ in 2030 [1]. While the increasing prevalence of type 2 diabetes is mainly due to the obesity epidemic, obesity is also associated with obstructive sleep apnoea (OSA) [2].

OSA is characterised by repetitive episodes of upper airway collapse during sleep, which cause enhanced sympathetic activity, increased cytokine levels, elevated oxidative stress and sleep fragmentation [3]. Recently, numerous studies have reported cross-sectional associations between OSA and type 2 diabetes, impaired glucose metabolism and insulin resistance [4, 5]. The Sleep Heart Health Study showed that the prevalence of type 2 diabetes was twofold higher among persons with OSA than in those without it and was independent of BMI and waist circumference [6]. Similarly, the Wisconsin Sleep Cohort Study showed a cross-sectional association between OSA and type 2 diabetes [7]. The investigators also suggested a longitudinal association between OSA and risk of type 2 diabetes, but it was not significant after adjustment for waist circumference [7]. Recently, although a longitudinal association between OSA and incident type 2 diabetes has been shown in Australians, the CIs were very wide, with multivariable-adjusted odds ratios (95\% CI) adjusted for age, sex and waist circumference of 8.62 (1.14-65.20) [8].

Since the longitudinal evidence on the association between OSA and type 2 diabetes is limited, we examined the association between nocturnal intermittent hypoxia as a surrogate marker of OSA and the risk of developing type 2 diabetes among community-dwelling Japanese participants.

\section{Methods}

Study overview The Circulatory Risk in Communities Study (CIRCS) is a prospective community-based study that was launched to prevent stroke in five communities across Japan from 1963 onwards and has been described in detail elsewhere [9]. Annual cardiovascular risk surveys and monitoring of cardiovascular events were systematically conducted by a research team of the Osaka Medical Center for Health Science and Promotion, the Osaka University and the University of Tsukuba. Investigation of sleep in the annual cardiovascular surveys was started in three communities (Ikawa, a north-eastern rural community; Yao, a midwestern suburban community; and Kyowa, a mid-eastern rural community) in 2000 [10]. The study protocol was approved by the Institutional Review Board of the Osaka
Medical Center for Health Science and Promotion, the Osaka University and the University of Tsukuba.

Participants Participants aged 40 to 69 years in the 2001 to 2005 annual cardiovascular surveys were included in the present investigation. A total of 448 men and 853 women from Yao, 397 men and 571 women from Ikawa, and 917 men and 1,507 women from Kyowa were eligible for this study. Data for sleep were available for $94.2 \%$ of participants aged 40 to 69 years in the annual cardiovascular survey. None of the participants had a history of OSA diagnosed by a physician, probably because that disorder had not been recognised as such by general physicians. We excluded participants with a history of stroke $(n=55)$ and heart disease $(n=33)$, and those with type 1 or type 2 diabetes (fasting serum glucose $\geq 7.00 \mathrm{mmol} / 1(126 \mathrm{mg} / \mathrm{dl})$ or non-fasting serum glucose $\geq 11.1 \mathrm{mmol} / \mathrm{l}(200 \mathrm{mg} / \mathrm{dl})$ and/or on glucose-lowering medication or insulin) at baseline $(n=278)$. A total of 1,603 men and 2,795 women were enrolled in the present study. A physician or trained staff explained the protocol in detail to each participant and obtained informed consent.

Assessment of nocturnal intermittent hypoxia Nocturnal intermittent hypoxia was measured by pulse-oximetry during one night of sleep using a pulse-oximeter (PULSOX-3Si; Minolta, Osaka, Japan) at the participant's own home [10]. The sensor probe was fitted to the fourth or fifth finger with a tape. The device stores the values of peripheral blood oxygen saturation $\left(\mathrm{SpO}_{2}\right)$ by performing a moving average for the last 5 seconds, updated every second; this sampling time was short enough to avoid underestimation of oxygen desaturation [11]. Data were downloaded to a personal computer via an interface (PULSOX IF-3; Minolta) and analysed by proprietary software (DS-3 version 2.0a; Minolta). A desaturation event began with a saturation decrease of $\geq 3 \%$ at intervals of 8 to $120 \mathrm{~s}$ and terminated when the saturation rose within $20 \mathrm{~s}$ by the appropriate percentage above the lowest saturation recorded during that event. Because the measurement time of pulse-oximetry is often longer than the true total sleep time, we used single night sleep log to exclude waking time from the analysis to minimise the potential for overestimating total sleep time. We used the number of oxygen desaturation measurements of $3 \%$ or more per $h$ of the adjusted measurement time $(3 \%$ oxygen desaturation index [3\%ODI]) as the indicator of nocturnal intermittent hypoxia. The severity of nocturnal intermittent hypoxia was defined by the $3 \%$ ODI level as five or 15 events per $h$, corresponding to mild or moderate-to-severe nocturnal intermittent hypoxia, respectively. Similarly, we used lowest $\mathrm{SpO}_{2}$ and duration of $\mathrm{SpO}_{2}<90 \%$ as the indicators for nocturnal hypoxia. 
The validity of pulse-oximetry has been previously confirmed by synchronous overnight recording with PULSOX-3Si and standard polysomnography among 256 consecutive patients in a sleep-disordered breathing centre (mean BMI, $26.8 \mathrm{~kg} / \mathrm{m}^{2}$ ). The sensitivity and specificity were $80 \%$ and $95 \%$, respectively, for detecting an apnoeahypopnoea index (AHI) of $\geq 5$ by polysomnography using a cut-off threshold of $3 \% \mathrm{ODI}=5$. Similarly, the sensitivity and specificity for detecting AHI of $\geq 20$ by polysomnography using a cut-off threshold of $3 \% \mathrm{ODI}=15$ were $85 \%$ and $100 \%$, respectively [12]. To examine reproducibility, pulseoximetry was conducted on two consecutive nights among 61 men in the present study. The median values of 3\%ODI were 5.4 on the first night and 4.8 on the second night ( $p=$ 0.95 for difference, Wilcoxon's signed-rank test). Spearman's rank correlation coefficient was $0.81(p<0.001)$.

Diagnosis of type 2 diabetes At the annual cardiovascular surveys, venous blood was drawn from seated participants into a plain, silicon-coated glass tube and serum was separated within $30 \mathrm{~min}$. The serum sample was transported on dry ice to the Osaka Medical Center for Health Science and Promotion and stored at $-70^{\circ} \mathrm{C}$ until measurement. Serum glucose was measured by an automatic analyser (AU2700; Olympus, Tokyo, Japan) based on the hexokinase method. The participants were recommended to have fasted overnight, but this was not necessary. The time since the last meal was recorded, with fasting defined as drawing blood more than $8 \mathrm{~h}$ after the last meal.

Monitoring after the sleep study was done at the annual cardiovascular surveys until the end of 2007. The development of type 2 diabetes was defined as: (1) fasting serum glucose $\geq 7.00 \mathrm{mmol} / 1(126 \mathrm{mg} / \mathrm{dl})$; (2) non-fasting serum glucose $\geq 11.1 \mathrm{mmol} / 1$ (200 mg/dl); and/or (3) initiation of treatment with glucose-lowering medication or insulin.

Measurement of potential confounding variables We measured several potential confounders that might contribute to the development of OSA and type 2 diabetes [2, 13]. BMI was calculated as weight in light clothing $(\mathrm{kg})$ divided by square of height in stocking feet (m). Waist circumference at the level of the umbilicus in a standing position with normal breathing was measured to the nearest $1 \mathrm{~cm}$ with a tape measure from 2003 in Kyowa and from 2006 in Ikawa and Yao. Trained public health nurses interviewed the participants about drinking and smoking habits, the frequency of habitual snoring and excessive daytime sleepiness, use of glucose-lowering medication or insulin, and (for women) menopausal status. The usual weekly alcohol intake was converted to grams of ethanol per day. Persons who smoked $\geq 1$ cigarette/day and those who stopped smoking were defined as current and former smokers, respectively. Persons who snored $\geq 5$ days a week and those who had excessive daytime sleepiness $\geq 5$ days a week were defined as habitual snorers and as having excessive daytime sleepiness, respectively. The presence or absence of borderline diabetes was defined as (1) fasting serum glucose $\geq 6.11 \mathrm{mmol} / \mathrm{l}(110 \mathrm{mg} / \mathrm{dl})$ or (2) non-fasting serum glucose $\geq 7.77 \mathrm{mmol} / 1(140 \mathrm{mg} / \mathrm{dl})$.

In the present study, we use single night sleep duration under pulse-oximetry as a surrogate of usual sleep duration. We examined the validity of equating usual sleep duration to single night sleep duration under pulse-oximetry among 172 women in the present study. Mean values of usual sleep duration and single night sleep duration were $7 \mathrm{~h} 3 \mathrm{~min}$ and 7 h $5 \mathrm{~min}$, respectively ( $p=0.75$ for difference, paired $t$ test). Pearson's correlation coefficient was $0.57(p<0.001)$.

Statistical analysis The person-years of follow-up were calculated as the time from the baseline survey date to the survey date when the patient was newly diagnosed as having type 2 diabetes or to the last follow-up. Participants with follow-up $\geq 1$ year were included in analyses. We categorised lowest $\mathrm{SpO}_{2}$ into quartiles and duration of $\mathrm{SpO}_{2}<90 \%$ into zero duration and tertiles for participants with $>0 \%$ duration of $\mathrm{SpO}_{2}<90 \%$. Univariable association of $3 \% \mathrm{ODI}$, lowest $\mathrm{SpO}_{2}$ and duration of $\mathrm{SpO}_{2}<90 \%$ at baseline with the development of type 2 diabetes was tested by logrank tests with Kaplan-Meier curves. Age, sexadjusted and multivariable-adjusted hazard ratios $(95 \% \mathrm{CI})$ for the development of type 2 diabetes according to $3 \%$ ODI, lowest $\mathrm{SpO}_{2}$ and duration of $\mathrm{SpO}_{2}<90 \%$ at baseline were calculated and tested by Cox's proportional hazards model. The assumption of proportional hazards was tested by the inclusion of time-dependent variables in the model and the assumption was not rejected. Trend tests were performed using regression models for characteristics and using Cox's proportional-hazards model, with median values of $3 \% \mathrm{ODI}$ at baseline as a representative value for nocturnal intermittent hypoxia severity. We also examined the dose-response relation between nocturnal intermittent hypoxia and development of type 2 diabetes by restricted cubic spline hazard regression models with knots placed at the 5th, 33rd, 66th and 95th percentile, and 3\%ODI values of $0.19,1.33,3.77$ and 13.8, and lowest $\mathrm{SpO}_{2}$ values of 73, 86, 91 and 94 [14]. References were selected at the 1st percentile, with zero events $/ \mathrm{h}$ for $3 \% \mathrm{ODI}$ and $95 \%$ for lowest $\mathrm{SpO}_{2}$. We did not graph predictions from the top and bottom $1 \%$ of the analytical distribution to avoid undue visual influence of sparse tail data. The interactions of 3\% ODI with sex and BMI in relation to the incidence of type 2 diabetes were tested using cross-product terms of these variables in Cox proportional hazards model. We included baseline data of age (year), sex, BMI $\left(\mathrm{kg} / \mathrm{m}^{2}\right)$ and community in the multivariable model (Model 1). Additional analyses were further adjusted for smoking status 
(never, former and current smoker), current alcohol intake (g/day), the presence or absence of borderline type 2 diabetes (yes and no), single night sleep duration $(<6,7$ and $\geq 8 \mathrm{~h}$ ), and (for women) menopausal status (pre- and post-menopause) (Model 2). Single night sleep duration was regarded as a surrogate of usual sleep duration because we found that these two variables were correlated strongly $(r=0.57)$, taken into account the large inter-night variation in sleep duration among normal sleepers [15]. All analyses were conducted using SAS statistical package version 9.13 (SAS Institute, Cary, NC, USA). All $p$ values for statistical tests were two-tailed and $p<0.05$ was regarded as statistical significance.

\section{Results}

By the end of 2007, 92.2\% of participants had been followed up (median follow-up duration [interquartile range] 3.0 [2.9-4.0] years), and 118 men and 92 women found to have newly diagnosed type 2 diabetes. The proportion of persons lost to the follow-up did not vary according to $3 \% \mathrm{ODI}$ : $9.5 \%$ for no nocturnal intermittent hypoxia, $8.3 \%$ for mild and $7.7 \%$ for moderate-to-severe nocturnal intermittent hypoxia among men; $7.2 \%, 6.7 \%$ and $6.5 \%$ respectively among women. The sex-specific ageadjusted prevalence of mild and moderate-to-severe nocturnal intermittent hypoxia was comparable among the three communities; men $36.2 \%$ for mild and $9.1 \%$ for moderate-to-severe nocturnal intermittent hypoxia in Ikawa, $30.8 \%$ and $9.2 \%$ in Yao, and $29.8 \%$ and $7.9 \%$ in Kyowa; women $17.5 \%$ and $2.0 \%$ in Ikawa, $14.6 \%$ and $3.1 \%$ in Yao, and $16.0 \%$ and $2.0 \%$ in Kyowa, respectively. The risk characteristics at baseline for the participants are summarised in Table 1.

Type 2 diabetes-free Kaplan-Meier survival curves differed significantly by the categories 3\%ODI, lowest $\mathrm{SpO}_{2}$ and duration of $\mathrm{SpO}_{2}<90 \%(p<0.001, p<0.001$ and $p=0.02$, respectively, logrank test) (Fig. 1a-c). Compared with the no nocturnal intermittent hypoxia group, the development of type 2 diabetes was 1.5 -fold higher among participants with mild nocturnal intermittent hypoxia $(3 \%$ ODI 5.0-14.9) and 2.5-fold higher among those with moderate-to-severe nocturnal intermittent hypoxia $(3 \%$ $\mathrm{ODI} \geq 15$ ) with a dose-response relationship discernible (Table 2). The development of type 2 diabetes was threefold higher among persons with lowest $\mathrm{SpO}_{2}<92 \%$ than in those with lowest $\mathrm{SpO}_{2} \geq 92 \%$, but the association was not in a graded fashion. Duration of $\mathrm{SpO}_{2}<90 \%$ was not associated with risk of type 2 diabetes. After adjustment for age, sex, BMI and community, these associations became weaker, but the trend did not change essentially. Moreover, further adjustment for smoking and drinking habits, the presence or absence of borderline diabetes, sleep duration and menopausal status did not markedly change the magnitude of association.

The shape of the dose-response curve is shown in Fig. 2a, b, using restricted cubic spline hazard regression models and adjusting for the full set of covariates. The results from categorical analysis are largely supported by these graphs. The hazard ratios for developed diabetes appear to linearly increase according to 3\%ODI and appear to increase within the lowest $\mathrm{SpO}_{2}$ range 89 to $95 \%$, and to plateau thereafter. However, the inference on the doseresponse pattern at the right tail is based on limited amount of data.
Table 1 Baseline risk characteristics of participants

Values are mean (SD), median (interquartile range) $^{\mathrm{a}}$ and proportions

\begin{tabular}{|c|c|c|c|}
\hline Characteristic & Overall & Men & Women \\
\hline$n$ & 3,864 & 1,341 & 2,523 \\
\hline \multicolumn{4}{|l|}{ Demographic } \\
\hline Age (years) & $57.6(8.1)$ & $58.3(7.8)$ & $56.6(7.8)$ \\
\hline Women $(\%)$ & 65.3 & - & - \\
\hline Post-menopause (\%) & 49.0 & - & 75.1 \\
\hline BMI $\left(\mathrm{kg} / \mathrm{m}^{2}\right)$ & $23.5(3.2)$ & $24.0(2.9)$ & $23.3(3.3)$ \\
\hline Current alcohol intake $(\mathrm{g} / \text { day })^{\mathrm{a}}$ & $0.0(0.0-9.9)$ & $19.7(0.0-46.0)$ & $0.0(0.0-0.0)$ \\
\hline Current smoker, $\%$ & 17.2 & 41.0 & 4.5 \\
\hline Borderline diabetes $(\%)$ & 6.0 & 9.3 & 4.2 \\
\hline \multicolumn{4}{|l|}{ Sleep characteristic } \\
\hline Habitual snoring (\%) & 16.5 & 28.3 & 10.2 \\
\hline Excessive daytime sleepiness (\%) & 4.6 & 6.6 & 3.6 \\
\hline $3 \%$ ODI (events $/ \mathrm{h})^{\mathrm{a}}$ & $2.3(1.0-5.0)$ & $2.3(1.0-5.0)$ & $1.8(0.8-3.8)$ \\
\hline Lowest $\mathrm{SpO}_{2}(\%)^{\mathrm{a}}$ & $88(84-91)$ & $87(82-90)$ & $89(85-92)$ \\
\hline Duration of $\mathrm{SpO}_{2}<90 \%(\%)^{\mathrm{a}}$ & $0.05(0.00-0.53)$ & $0.16(0.00-1.04)$ & $0.02(0.00-0.30)$ \\
\hline Sleep duration $(\mathrm{h})^{\mathrm{a}}$ & $7.2(6.3-8.0)$ & $7.6(6.7-8.5)$ & $7.0(6.1-7.8)$ \\
\hline
\end{tabular}



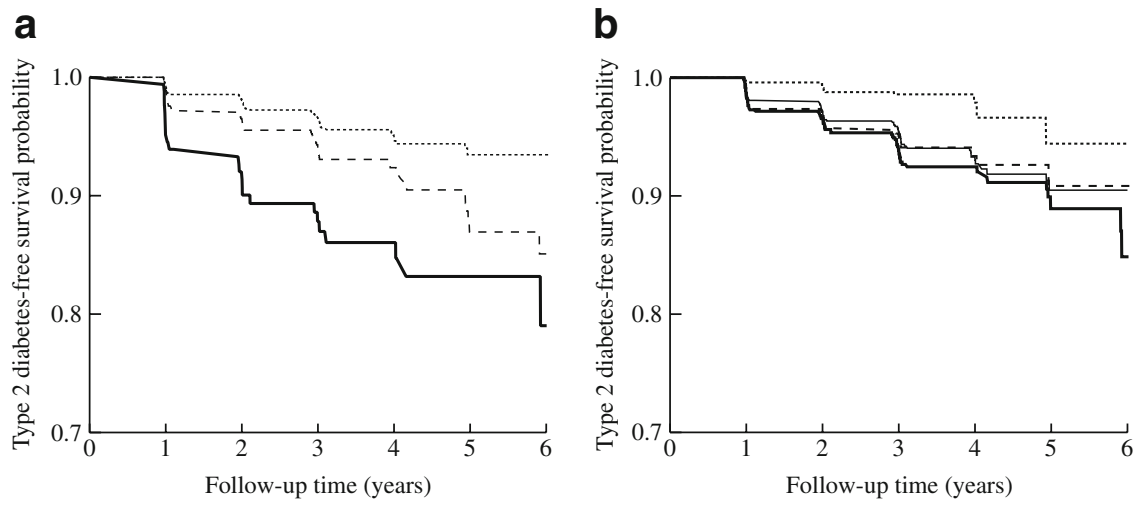

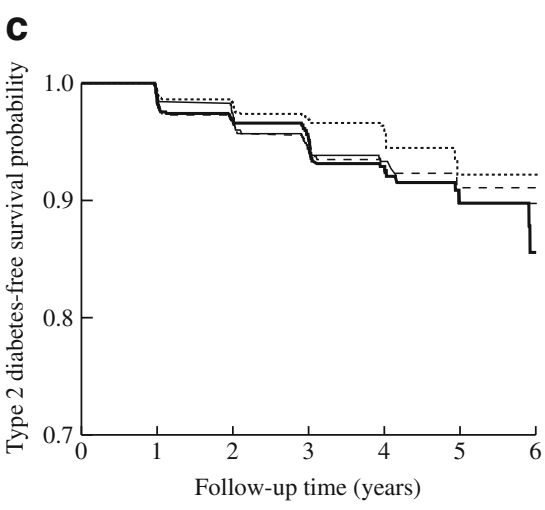

Fig. 1 Type 2 diabetes free-survival among 4,398 participants, stratified by $3 \% \mathrm{ODI}$ (a) (dotted line, 3\%ODI $<5.0$; dashed line, 3\% ODI 5.0-14.9; continuous line, $3 \%$ ODI $\geq 15$ [ $p<0.001$, logrank test]), lowest $\mathrm{SpO}_{2}$ (b) (dotted line, $\mathrm{SpO}_{2} \geq 92 \%$; dashed line, $\mathrm{SpO}_{2} 89-91 \%$;

Because abdominal obesity was shown to be associated more strongly than BMI for development of type 2 diabetes, we restricted our analysis to the data from Kyowa since 2003 ( $n=1,404$ with 57 cases of type 2 diabetes) as subgroup analysis. The risk of type 2 diabetes was 1.99 (95\% CI $1.08-3.66)$ at $3 \%$ ODI 5 to $<15$ and $2.83(0.99$ 8.09 ) at $3 \% \mathrm{ODI} \geq 15$ after adjustment for waist circumference and other confounding factors, compared with persons with no nocturnal intermittent hypoxia $(p=0.01$ for trend). In addition, the risk of type 2 diabetes was 4.14 thin line, $\mathrm{SpO}_{2}$ 84-88\%; bold line, $\left.\mathrm{SpO}_{2} \leq 83 \%[p<0.001]\right)$ and duration of $\mathrm{SpO}_{2}<90 \%$ (c) (dotted line, $\mathrm{SpO}_{2}<90 \% 0 \%$; dashed line, $\mathrm{SpO}_{2}<90 \% 0.01-0.12 \%$; thin line, $\mathrm{SpO}_{2}<90 \% 0.12-0.79 \%$; bold line, $\left.\mathrm{SpO}_{2}<90 \% \geq 0.80 \%[p=0.01]\right)$

(1.19-14.4) with lowest $\mathrm{SpO}_{2} 89$ to $91 \%, 3.29(0.94-11.6)$ with lowest $\mathrm{SpO}_{2} 84$ to $88 \%$ and 4.44 (1.26-15.7) with lowest $\mathrm{SpO}_{2} \leq 83 \%$, compared with lowest $\mathrm{SpO}_{2} \geq 92 \%$ ( $p=0.08$ for trend). For duration of $\mathrm{SpO}_{2}$, the risk of type 2 diabetes was $1.51(0.73-3.13)$ for duration of $\mathrm{SpO}_{2}<$ $90 \%=0.01-0.12 \%, 1.26(0.58-2.72)$ for duration of $\mathrm{SpO}_{2}<$ $90 \%=0.13-0.79 \%$ and $1.63(0.77-3.43)$ for duration of $\mathrm{SpO}_{2}<90 \% \geq 0.80 \%$, compared with duration of $\mathrm{SpO}_{2}<$ $90 \%=0 \%$ ( $p=0.34$ for trend). Nevertheless, that hazard ratio estimate was similar to that after adjustment for BMI

Table 2 Hazard ratios of type 2 diabetes according to $3 \%$ ODI, lowest $\mathrm{SpO}_{2}$ and duration of $\mathrm{SpO}_{2}<90 \%$ at baseline

\begin{tabular}{|c|c|c|c|c|c|}
\hline Variable & Person-years & Developed diabetes $(n)$ & Age, sex-adjusted HR & Model $1 \mathrm{HR}$ & Model 2 HR \\
\hline \multicolumn{6}{|l|}{$3 \% O D I$ (events/h) } \\
\hline$<5$ & 9,545 & 117 & (Reference) & (Reference) & (Reference) \\
\hline $5-14.9$ & 3,092 & 68 & $1.54(1.13-2.10)$ & $1.20(0.87-1.66)$ & $1.26(0.91-1.76)$ \\
\hline$\geq 15$ & 655 & 25 & $2.45(1.56-3.83)$ & $1.65(1.02-2.66)$ & $1.69(1.04-2.76)$ \\
\hline$p$ value for trend & & & $<0.001$ & 0.04 & 0.03 \\
\hline \multicolumn{6}{|l|}{ Lowest $\mathrm{SpO}_{2}$} \\
\hline$\geq 92 \%$ & 2,344 & 12 & (Reference) & (Reference) & (Reference) \\
\hline $89-91 \%$ & 2,816 & 48 & $2.94(1.56-5.54)$ & $2.68(1.42-5.06)$ & $2.40(1.27-4.55)$ \\
\hline $84-88 \%$ & 3,724 & 64 & $2.68(1.44-5.01)$ & $2.26(1.20-4.22)$ & $2.06(1.09-3.88)$ \\
\hline$\leq 83 \%$ & 3,047 & 64 & $3.18(1.70-5.94)$ & $2.36(1.25-4.46)$ & $2.12(1.12-4.03)$ \\
\hline$p$ value for trend & & & 0.008 & 0.21 & 0.34 \\
\hline \multicolumn{6}{|l|}{ Duration of $\mathrm{SpO}_{2}<90 \%$} \\
\hline $0 \%$ of sleep duration & 4,656 & 49 & (Reference) & (Reference) & (Reference) \\
\hline $0.01-0.12 \%$ of sleep duration & 2,613 & 47 & $1.48(0.99-2.21)$ & $1.37(0.91-2.05)$ & $1.25(0.83-1.89)$ \\
\hline $0.13-0.79 \%$ of sleep duration & 2,717 & 49 & $1.40(0.94-2.10)$ & $1.19(0.79-1.79)$ & $1.17(0.78-1.75)$ \\
\hline$\geq 0.80 \%$ of sleep duration & 2,772 & 53 & $1.39(0.93-2.07)$ & $1.02(0.67-1.55)$ & $0.95(0.63-1.44)$ \\
\hline$p$ value for trend & & & 0.49 & 0.43 & 0.31 \\
\hline
\end{tabular}

Unless otherwise indicated, values are HR $(95 \% \mathrm{CI})$

Model 1 was adjusted for age (years), sex, BMI $\left(\mathrm{kg} / \mathrm{m}^{2}\right)$ and community; Model 2 was adjusted for factors cited above and smoking status (never, former, current), current alcohol intake (g/day), borderline diabetes (yes/no), single night sleep duration $(<6,7, \geq 8 \mathrm{~h})$ and (for women) menopausal status (pre-, post-menopause) 


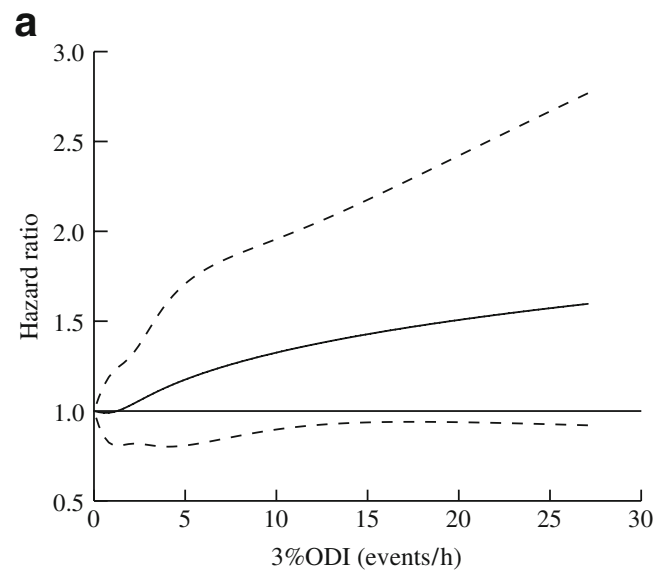

b

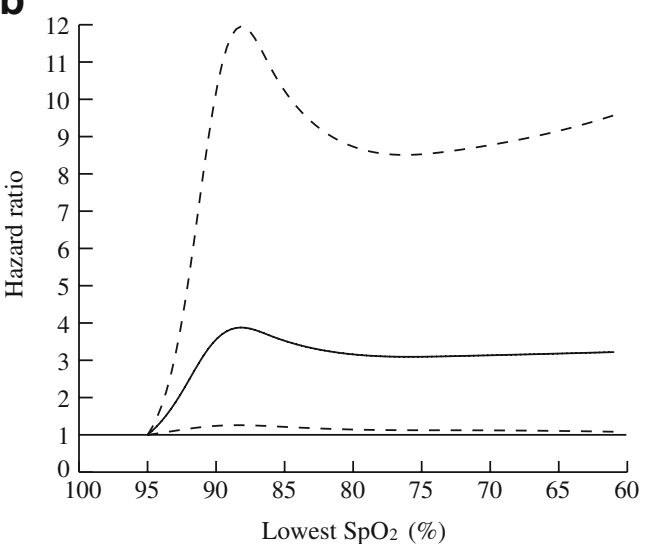

Fig. 2 Restricted cubic spline hazard regression of developed type 2 diabetes on the $3 \% \mathrm{ODI}$ (a) and lowest $\mathrm{SpO}_{2}$ (b). Knots were placed at the 5 th, 33rd, 66th and 95th percentile. Continuous lines, predicted HRs; dashed lines, 95\% CIs. To avoid undue visual influence of sparse tail data, we did not graph predictions from the top and bottom $1 \%$ of the analytical distribution

and other confounding factors $(p=0.03$ for trend for $3 \%$ ODI, $p=0.11$ for trend for lowest $\mathrm{SpO}_{2}$ and $p=0.43$ for trend for duration of $\mathrm{SpO}_{2}<90 \%$, data not shown in table).

\section{Discussion}

Our prospective study showed that nocturnal intermittent hypoxia and lowest $\mathrm{SpO}_{2}$ during the night are associated with increased risk of type 2 diabetes independently of its traditional risk factors such as age, sex and BMI. These associations were not confounded by smoking and drinking habits, the presence or absence of borderline diabetes, single night sleep duration and (for women) menopausal status.

In the Sleep Heart Health Study $(n=6,441)$, the prevalence of type 2 diabetes assessed by $75 \mathrm{~g}$ oral glucose tolerance test was 1.5 to 1.7 -fold higher among persons with $\geq 10$ events/h respiratory disturbance index (RDI), an indicator of OSA defined in a similar way to AHI, than among those with RDI $<10$ as assessed by polysomnography at home [6]. Similarly in the Wisconsin Sleep Cohort Study $(n=1,387)$, odds ratio of prevalent diabetes defined by self-reported physician's diagnosis was 2.30 (95\% CI 1.28-4.11) for $\mathrm{AHI} \geq 15$ vs $\mathrm{AHI}<5$ as assessed by polysomnography in laboratory [7]. When the investigators also examined a longitudinal association between OSA and 4-year incidence of type 2 diabetes, a weak and insignificant association was observed, with odds ratios of 1.56 (95\% CI $0.80-3.02)$ for AHI 5 to $<15$ and $1.62(0.67-3.65)$ for AHI $\geq 15$ after adjustment for age, sex and waist circumference [7]. Recently, the Busselton Health Study, an Australian cohort study $(n=295)$, showed a significant association between OSA assessed by four-channel portable monitoring device (Mesam4; Madaus Medizin-Elektronik, Freiberg, Germany) [16] at home and incident diabetes defined by physician's diagnosis or fasting blood glucose $\geq 7.00 \mathrm{mmol} / 1$, although the number for incident diabetes was small $(n=9)$; odds ratio was $1.31(95 \%$ CI $0.23-7.35)$ for RDI 5 to $<15$ and $8.62(1.14-65.20)$ for RDI $\geq 15$, compared with RDI $<5$ after adjustment for age, sex and waist circumference [8]. Because our study measured 3\% ODI by pulse-oximetry only, our results may not be directly comparable with the results from the two previous cohort studies, which used polysomnography in laboratory settings or four-channel portable monitoring devices at home. However, since pulse-oximetry has been successfully validated [12], our results are likely to confirm the previous findings.

The mechanisms involved in the associations between nocturnal intermittent hypoxia and development of type 2 diabetes may be hypoxia and oxidative stress due to repetitive hypoxia and re-oxygenation. First, reduced blood oxygen saturation stimulates sympathetic nerves mediated by peripheral arterial chemoreceptors, particularly carotid bodies, with the enhanced sympathetic drive apparently being carried over into normal waking hours [17]. Enhanced sympathetic drive was shown to raise serum catecholamine levels, leading to elevated serum glucose levels [18]. Second, elevated oxidative stress raises cytokine levels (e.g. TNF- $\alpha$ ) [19], leading to increased insulin resistance [20]. Third, sleep fragmentation was found to activate the hypothalamic-pituitary-adrenal axis [21]. The activation of this axis causes a marked elevation in serum cortisol levels, leading to insulin resistance and hyperglycaemia [22].

The strengths of our study include a prospective cohort design with a large sample size and a significant number of participants with incident type 2 diabetes. A second strength is that we assessed type 2 diabetes by standardised and objective methods. Third, we adjusted for potential confounding variables, e.g. smoking and drinking habits, 
menopausal status, sleep duration and the presence or absence of borderline diabetes, as well as age, sex and BMI. In particular, borderline diabetes and sleep duration were recently noted to be predictors of future type 2 diabetes $[23,24]$.

Our study also has several limitations. First, we only measured oxygen desaturation during sleep by pulse-oximetry as a way to estimate nocturnal intermittent hypoxia. The sensitivity of $3 \% \mathrm{ODI} \geq 5$ when screening for $\mathrm{AHI} \geq 5$ and of $3 \% \mathrm{ODI} \geq 15$ when screening for $\mathrm{AHI} \geq 20$ was $68 \%$ and $76 \%$ among participants with $\mathrm{BMI} \leq 27.0 \mathrm{~kg} / \mathrm{m}^{2}$ and $94 \%$ and $93 \%$ among those with BMI $>27.0 \mathrm{~kg} / \mathrm{m}^{2}$ [12]. Because our study population had low BMI (mean BMI $23.4 \mathrm{~kg} / \mathrm{m}^{2}$ ), pulseoximetry is not comparable to gold standard polysomnography, provides no information about sleep stage or body position, and could underestimate the degree of OSA. However, pulse-oximetry conducted during sleep at home has the advantage of reflecting the usual sleep position because the sleep time spent in supine position was 1.5fold longer during polysomnography than during nonpolysomnography [25]. The second limitation is that detection of central sleep apnoea and hypoventilation cannot be distinguished from OSA with pulse-oximetry. Diabetes mellitus is known to cause central-type intermittent hypoxia, but the frequency was only $5 \%$ in diabetes patients [26]. Thus, the contamination may be small. Third, as study participants were not interviewed about pre-existing lung disease, underlying chronic obstructive pulmonary diseases or asthma, which are associated with nocturnal hypoventilation or hypoxia, may have confounded results. According to the 2005 Japan National Patient Survey, only $0.1 \%$ of persons aged 40 to 69 years sought consultations due to these lung diseases [27]. Therefore, confounding due to lung diseases is likely to be minimum. The fourth limitation is that we only adjusted for BMI as a marker of body composition, but abdominal obesity has recently shown to be associated more strongly than BMI with type 2 diabetes [28]. In our study, waist circumference was measured systematically from 2003 in Kyowa and from 2006 in Yao and Ikawa. When we restricted our analysis to the data from Kyowa since 2003, the hazard risk estimates after adjustment for waist circumference and other confounding factors were similar to those for BMI. Last, the follow-up period is relatively short (2.9-4.0 years) in the present study. A longterm effect of nocturnal intermittent hypoxia on risk of type 2 diabetes will be explored in the future.

In summary, the present large cohort study showed explicitly that nocturnal intermittent hypoxia was associated with increased risk of developing type 2 diabetes among middle-aged Japanese.

Acknowledgements We thank M. Umesawa, Y. Ichikawa and M. Notsute for their technical assistance and M. Kudo, K. Kamei and staff members of the Osaka Medical Center for Health Science and Promotion, Osaka University and the University of Tsukuba for data collection. This study was supported in part by grants from the Japanese Ministry of Education, Culture, Sports, Science and Technology (Grant-in-Aid for research B: 14370132 and 18390194) and a Health and Laboratory Sciences Research Grant (Clinical Research for Evidence Based Medicine), as well as by the Ministry of Health, Welfare and Labor, the FULLHAP, Japan, and the University Research Project Research Grant (B), University of Tsukuba.

Duality of interest The authors declare that there is no duality of interest associated with this manuscript.

\section{References}

1. Wild S, Roglic G, Green A, Sicree R, King H (2004) Global prevalence of diabetes: estimates for the year 2000 and projections for 2030. Diabetes Care 27:1047-1053

2. Young T, Peppard PE, Gottlieb DJ (2002) Epidemiology of obstructive sleep apnea: a population health perspective. Am J Respir Crit Care Med 165:1217-1239

3. Caples SM, Garcia-Touchard A, Somers VK (2007) Sleepdisordered breathing and cardiovascular risk. Sleep 30:291-303

4. Shaw JE, Punjabi NM, Wilding JP, Alberti KG, Zimmet PZ, International Diabetes Federation Taskforce on Epidemiology and Prevention (2008) Sleep-disordered breathing and type 2 diabetes: a report from the International Diabetes Federation Taskforce on Epidemiology and Prevention. Diabetes Res Clin Pract 81:2-12

5. Punjabi NM, Shahar E, Redline S, Gottlieb DJ, Givelber R, Resnick HE, Sleep Heart Health Study Investigators (2004) Sleepdisordered breathing, glucose intolerance, and insulin resistance: the Sleep Heart Health Study. Am J Epidemiol 160:521-530

6. Seicean S, Kirchner HL, Gottlieb DJ et al (2008) Sleep-disordered breathing and impaired glucose metabolism in normal-weight and overweight/obese individuals: the Sleep Heart Health Study. Diabetes Care 31:1001-1006

7. Reichmuth KJ, Austin D, Skatrud JB, Young T (2005) Association of sleep apnea and type II diabetes: a population-based study. Am J Respir Crit Care Med 172:1590-1595

8. Marshall NS, Wong KK, Phillips CL, Liu PY, Knuiman MW, Grunstein RR (2009) Is sleep apnea an independent risk factor for prevalent and incident diabetes in the Busselton Health Study? J Clin Sleep Med 5:15-20

9. Imano H, Kitamura A, Sato S et al (2009) Trends for blood pressure and its contribution to stroke incidence in the middleaged Japanese population: the Circulatory Risk in Communities Study (CIRCS). Stroke 40:1571-1577

10. Tanigawa T, Tachibana N, Yamagishi K et al (2004) Usual alcohol consumption and arterial oxygen desaturation during sleep. JAMA 292:923-925

11. West SD, Nicoll DJ, Wallace TM, Matthews DR, Stradling JR (2007) Effect of CPAP on insulin resistance and HbA1c in men with obstructive sleep apnoea and type 2 diabetes. Thorax 62:969-974

12. Nakamata M, Kubota Y, Sakai K et al (2003) The limitation of screening test for patients with sleep apnea syndrome using pulse oximetry. J Jpn Soc Respir Care 12:401-406 (in Japanese)

13. Chan JC, Malik V, Jia W et al (2009) Diabetes in Asia: epidemiology, risk factors, and pathophysiology. JAMA 301: 2129-2140

14. Heinz H, Kaider A (1997) Gaining more flexibility in Cox proportional hazards regression models with cubic spline functions. Comp Methods Programs Biomed 54:201-208 
15. Means MK, Edinger JD, Glenn DM, Fins AI (2003) Accuracy of sleep perceptions among insomnia sufferers and normal sleepers. Sleep Med 4:285-296

16. Esnaola S, Durán J, Infante-Rivard C, Rubio R, Fernández A (1996) Diagnostic accuracy of a portable recording device (MESAM IV) in suspected obstructive sleep apnoea. Eur Respir J 9:2597-2605

17. Narkiewicz K, van de Borne PJ, Montano N, Dyken ME, Phillips BG, Somers VK (1998) Contribution of tonic chemoreflex activation to sympathetic activity and blood pressure in patients with obstructive sleep apnea. Circulation 97:943-945

18. Nonogaki K (2000) New insights into sympathetic regulation of glucose and fat metabolism. Diabetologia 43:533-549

19. Ciftci TU, Kokturk O, Bukan N, Bilgihan A (2004) The relationship between serum cytokine levels with obesity and obstructive sleep apnea syndrome. Cytokine 28:87-91

20. Uysal KT, Wiesbrock SM, Marino MW, Hotamisligil GS (1997) Protection from obesity-induced insulin resistance in mice lacking TNF-alpha function. Nature 389:610-614

21. Stamatakis K, Punjabi NM (2009) Effects of sleep fragmentation on glucose metabolism in normal subjects. Chest. doi:10.1378/ chest.09-0791
22. Follenius M, Brandenberger G, Bandesapt JJ, Libert JP, Ehrhart J (1992) Nocturnal cortisol release in relation to sleep structure. Sleep 15:21-27

23. Abdul-Ghani MA, Lyssenko V, Tuomi T, DeFronzo RA, Groop L (2009) Fasting vs postload plasma glucose concentration and the risk for future type 2 diabetes: results from the Botnia Study. Diabetes Care 32:281-286

24. Gottlieb DJ, Punjabi NM, Newman AB et al (2005) Association of sleep time with diabetes mellitus and impaired glucose tolerance. Arch Intern Med 165:863-867

25. Metersky ML, Castriotta RJ (1996) The effect of polysomnography on sleep position: possible implications on the diagnosis of positional obstructive sleep apnea. Respiration 63:283-287

26. Resnick HE, Redline S, Shahar E et al (2003) Diabetes and sleep disturbances: findings from the Sleep Heart Health Study. Diabetes Care 26:702-709

27. Ministry of Health, Labour and Welfare, Japan (2005) National Patient Survey. Available from www.mhlw.go.jp/english/database/ db-hss/ps.html, accessed 17 May 2009

28. Balkau B, Deanfield JE, Després JP et al (2007) International Day for the Evaluation of Abdominal Obesity (IDEA): a study of waist circumference, cardiovascular disease, and diabetes mellitus in 168, 000 primary care patients in 63 countries. Circulation 116:1942-1951 